\title{
Long range rapidity correlations and jet production in high energy nuclear collisions
}

B. I. Abelev, ${ }^{8}$ M. M. Aggarwal, ${ }^{30}$ Z. Ahammed, ${ }^{47}$ A. V. Alakhverdyants, ${ }^{17}$ B. D. Anderson, ${ }^{18}$ D. Arkhipkin, ${ }^{3}$ G. S. Averichev, ${ }^{17}$ J. Balewski, ${ }^{22}$ O. Barannikova, ${ }^{8}$ L. S. Barnby, ${ }^{2}$ J. Baudot, ${ }^{15}$ S. Baumgart,${ }^{52}$ D. R. Beavis, ${ }^{3}$ R. Bellwied,${ }^{50}$ F. Benedosso, ${ }^{27}$

M. J. Betancourt, ${ }^{22}$ R. R. Betts, ${ }^{8}$ A. Bhasin, ${ }^{16}$ A. K. Bhati, ${ }^{30}$ H. Bichsel,,${ }^{49}$ J. Bielcik,,${ }^{10}$ J. Bielcikova,${ }^{11}$ B. Biritz, ${ }^{6}$ L. C. Bland, ${ }^{3}$ I. Bnzarov, ${ }^{17}$ M. Bombara, ${ }^{2}$ B. E. Bonner, ${ }^{36}$ J. Bouchet, ${ }^{18}$ E. Braidot,${ }^{27}$ A. V. Brandin,,${ }^{25}$ E. Bruna, ${ }^{52}$ S. Bueltmann, ${ }^{29}$

T. P. Burton, ${ }^{2}$ M. Bystersky, ${ }^{11}$ X. Z. Cai ${ }^{40}$ H. Caines,,${ }^{52}$ M. Calderón de la Barca Sánchez, ${ }^{5}$ O. Catu, ${ }^{52}$ D. Cebra, ${ }^{5}$ R. Cendejas, ${ }^{6}$ M. C. Cervantes, ${ }^{42}$ Z. Chajecki, ${ }^{28}$ P. Chaloupka, ${ }^{11}$ S. Chattopadhyay, ${ }^{47}$ H. F. Chen, ${ }^{38}$ J. H. Chen, ${ }^{18}$ J. Y. Chen, ${ }^{51}$ J. Cheng, ${ }^{44}$ M. Cherney, ${ }^{9}$ A. Chikanian, ${ }^{52}$ K. E. Choi, ${ }^{34}$ W. Christie, ${ }^{3}$ R. F. Clarke, ${ }^{42}$ M. J. M. Codrington, ${ }^{42}$ R. Corliss, ${ }^{22}$ T. M. Cormier, ${ }^{50}$ M. R. Cosentino, ${ }^{37}$ J. G. Cramer, ${ }^{49}$ H. J. Crawford ${ }^{4}$ D. Das, ${ }^{5}$ S. Dash, ${ }^{12}$ M. Daugherity, ${ }^{43}$ L. C. De Silva, ${ }^{50}$ T. G. Dedovich, ${ }^{17}$ M. DePhillips, ${ }^{3}$ A. A. Derevschikov, ${ }^{32}$ R. Derradi de Souza, ${ }^{7}$ L. Didenko, ${ }^{3}$ P. Djawotho, ${ }^{42}$ S. M. Dogra, ${ }^{16}$ X. Dong, ${ }^{21}$ J. L. Drachenberg, ${ }^{42}$ J. E. Draper, ${ }^{5}$ J. C. Dunlop, ${ }^{3}$ M. R. Dutta Mazumdar, ${ }^{47}$ L. G. Efimov, ${ }^{17}$ E. Elhalhuli, ${ }^{2}$ M. Elnimr,,${ }^{50}$ J. Engelage, ${ }^{4}$ G. Eppley ${ }^{36}$ B. Erazmus,${ }^{41}$ M. Estienne ${ }^{41}$ L. Eun ${ }^{31}$ P. Fachini, ${ }^{3}$ R. Fatemi,${ }^{19}$ J. Fedorisin, ${ }^{17}$ A. Feng, ${ }^{51}$ P. Filip ${ }^{17}$ E. Finch,${ }^{52}$ V. Fine, ${ }^{3}$ Y. Fisyak,${ }^{3}$ C. A. Gagliardi, ${ }^{42}$ L. Gaillard, ${ }^{2}$ D. R. Gangadharan, ${ }^{6}$ M. S. Ganti, ${ }^{47}$ E. J. Garcia-Solis, ${ }^{8}$ A. Geromitsos ${ }^{41}$ F. Geurts, ${ }^{36}$ V. Ghazikhanian, ${ }^{6}$ P. Ghosh,${ }^{47}$ Y. N. Gorbunov, ${ }^{9}$ A. Gordon, ${ }^{3}$ O. Grebenyuk, ${ }^{21}$ D. Grosnick, ${ }^{46}$ B. Grube, ${ }^{34}$ S. M. Guertin, ${ }^{6}$ K. S. F. F. Guimaraes, ${ }^{37}$ A. Gupta, ${ }^{16}$ N. Gupta,${ }^{16}$ W. Guryn, ${ }^{3}$ B. Haag, ${ }^{5}$ T. J. Hallman, ${ }^{3}$ A. Hamed, ${ }^{42}$ J. W. Harris,${ }^{52}$ W. He ${ }^{14}$ M. Heinz,${ }^{52}$ S. Heppelmann, ${ }^{31}$ B. Hippolyte, ${ }^{15}$ A. Hirsch,,${ }^{33}$ E. Hjort, ${ }^{21}$ A. M. Hoffman, ${ }^{22}$

G. W. Hoffmann, ${ }^{43}$ D. J. Hofman, ${ }^{8}$ R. S. Hollis, ${ }^{8}$ H. Z. Huang, ${ }^{6}$ T. J. Humanic, ${ }^{28}$ L. Huo, ${ }^{42}$ G. Igo, ${ }^{6}$ A. Iordanova, ${ }^{8}$ P. Jacobs, ${ }^{21}$ W. W. Jacobs, ${ }^{14}$ P. Jakl, ${ }^{11}$ C. Jena, ${ }^{12}$ F. Jin ${ }^{40}$ C. L. Jones, ${ }^{22}$ P. G. Jones, ${ }^{2}$ J. Joseph,${ }^{18}$ E. G. Judd, ${ }^{4}$ S. Kabana,${ }^{41}$ K. Kajimoto, ${ }^{43}$ K. Kang, ${ }^{44}$ J. Kapitan, ${ }^{11}$ K. Kauder, ${ }^{8}$ D. Keane, ${ }^{18}$ A. Kechechyan, ${ }^{17}$ D. Kettler, ${ }^{49}$ V. Yu. Khodyrev, ${ }^{32}$ D. P. Kikola, ${ }^{21}$ J. Kiryluk, ${ }^{21}$ A. Kisiel, ${ }^{48}$ S. R. Klein, ${ }^{21}$ A. G. Knospe, ${ }^{52}$ A. Kocoloski, ${ }^{22}$ D. D. Koetke, ${ }^{46}$ J. Konzer, ${ }^{33}$ M. Kopytine, ${ }^{18}$ I. Koralt, ${ }^{29}$ W. Korsch, ${ }^{19}$ L. Kotchenda, ${ }^{25}$ V. Kouchpil, ${ }^{11}$ P. Kravtsov, ${ }^{25}$ V. I. Kravtsov, ${ }^{32}$ K. Krueger, ${ }^{1}$ M. Krus,${ }^{10}$ C. Kuhn, ${ }^{15}$ L. Kumar, ${ }^{30}$ P. Kurnadi, ${ }^{6}$ M. A. C. Lamont, ${ }^{3}$ J. M. Landgraf, ${ }^{3}$ S. LaPointe, ${ }^{50}$ J. Lauret, ${ }^{3}$ A. Lebedev,${ }^{3}$ R. Lednicky, ${ }^{17}$ C.-H. Lee, ${ }^{34}$ J. H. Lee ${ }^{3}$ W. Leight, ${ }^{22}$ M. J. LeVine, ${ }^{3}$ C. Li,${ }^{38}$ N. Li, ${ }^{51}$ Y. Li ${ }^{44}$ G. Lin, ${ }^{52}$ S. J. Lindenbaum, ${ }^{26}$ M. A. Lisa, ${ }^{28}$ F. Liu, ${ }^{51}$ H. Liu, ${ }^{5}$ J. Liu, ${ }^{36}$ L. Liu ${ }^{51}$ T. Ljubicic, ${ }^{3}$ W. J. Llope, ${ }^{36}$ R. S. Longacre, ${ }^{3}$ W. A. Love, ${ }^{3}$ Y. Lu ${ }^{38}$ T. Ludlam, ${ }^{3}$ G. L. Ma, ${ }^{40}$ Y. G. Ma ${ }^{40}$ D. P. Mahapatra, ${ }^{12}$ R. Majka, ${ }^{52}$ O. I. Mall, ${ }^{5}$ L. K. Mangotra, ${ }^{16}$ R. Manweiler, ${ }^{46}$ S. Margetis, ${ }^{18}$ C. Markert, ${ }^{43}$ H. Masui, ${ }^{21}$ H. S. Matis, ${ }^{21}$ Yu. A. Matulenko, ${ }^{32}$ D. McDonald, ${ }^{36}$ T. S. McShane, ${ }^{9}$ A. Meschanin,,${ }^{32}$ R. Milner, ${ }^{22}$ N. G. Minaev, ${ }^{32}$ S. Mioduszewski, ${ }^{42}$ A. Mischke, ${ }^{27}$ B. Mohanty, ${ }^{47}$ D. A. Morozov, ${ }^{32}$ M. G. Munhoz,${ }^{37}$ B. K. Nandi, ${ }^{13}$ C. Nattrass, ${ }^{52}$ T. K. Nayak, ${ }^{47}$ J. M. Nelson, ${ }^{2}$ P. K. Netrakanti, ${ }^{33}$ M. J. Ng, ${ }^{4}$ L. V. Nogach ${ }^{32}$ S. B. Nurushev, ${ }^{32}$ G. Odyniec, ${ }^{21}$ A. Ogawa, ${ }^{3}$ H. Okada, ${ }^{3}$ V. Okorokov, ${ }^{25}$ D. Olson,,${ }^{21}$ M. Pachr, ${ }^{10}$ B. S. Page,${ }^{14}$ S. K. Pal,${ }^{47}$ Y. Pandit,${ }^{18}$ Y. Panebratsev, ${ }^{17}$ T. Pawlak ${ }^{48}$ T. Peitzmann, ${ }^{27}$ V. Perevoztchikov, ${ }^{3}$ C. Perkins, ${ }^{4}$ W. Peryt, ${ }^{48}$ S. C. Phatak, ${ }^{12}$ P. Pile, ${ }^{3}$ M. Planinic,,${ }^{53}$ M. A. Ploskon, ${ }^{21}$ J. Pluta, ${ }^{48}$ D. Plyku ${ }^{29}$ N. Poljak ${ }^{53}$ A. M. Poskanzer ${ }^{21}$ B. V. K. S. Potukuchi, ${ }^{16}$ D. Prindle,${ }^{49}$ C. Pruneau, ${ }^{50}$ N. K. Pruthi, ${ }^{30}$ P. R. Pujahari, ${ }^{13}$ J. Putschke, ${ }^{52}$ R. Raniwala, ${ }^{35}$ S. Raniwala, ${ }^{35}$ R. L. Ray, ${ }^{43}$ R. Redwine, ${ }^{22}$ R. Reed, ${ }^{5}$ A. Ridiger,${ }^{25}$ H. G. Ritter, ${ }^{21}$ J. B. Roberts ${ }^{36}$

O. V. Rogachevskiy, ${ }^{17}$ J. L. Romero, ${ }^{5}$ A. Rose, ${ }^{21}$ C. Roy, ${ }^{41}$ L. Ruan, ${ }^{3}$ M. J. Russcher, ${ }^{27}$ R. Sahoo, ${ }^{41}$ S. Sakai, ${ }^{6}$ I. Sakrejda,${ }^{21}$ T. Sakuma, ${ }^{22}$ S. Salur, ${ }^{21}$ J. Sandweiss, ${ }^{52}$ M. Sarsour, ${ }^{42}$ J. Schambach, ${ }^{43}$ R. P. Scharenberg, ${ }^{33}$ N. Schmitz, ${ }^{23}$ J. Seger, ${ }^{9}$ I. Selyuzhenkov, ${ }^{14}$ P. Seyboth, ${ }^{23}$ A. Shabetai, ${ }^{15}$ E. Shahaliev, ${ }^{17}$ M. Shao, ${ }^{38}$ M. Sharma, ${ }^{50}$ S. S. Shi,,${ }^{51}$ X.-H. Shi, ${ }^{40}$

E. P. Sichtermann, ${ }^{21}$ F. Simon, ${ }^{23}$ R. N. Singaraju, ${ }^{47}$ M. J. Skoby, ${ }^{33}$ N. Smirnov, ${ }^{52}$ P. Sorensen, ${ }^{3}$ J. Sowinski,,${ }^{14}$ H. M. Spinka, ${ }^{1}$ B. Srivastava, ${ }^{33}$ T. D. S. Stanislaus, ${ }^{46}$ D. Staszak, ${ }^{6}$ M. Strikhanov ${ }^{25}$ B. Stringfellow, ${ }^{33}$ A. A. P. Suaide, ${ }^{37}$ M. C. Suarez, ${ }^{8}$ N. L. Subba, ${ }^{18}$ M. Sumbera, ${ }^{11}$ X. M. Sun, ${ }^{21}$ Y. Sun, ${ }^{38}$ Z. Sun, ${ }^{20}$ B. Surrow, ${ }^{22}$ T. J. M. Symons, ${ }^{21}$ A. Szanto de Toledo, ${ }^{37}$

J. Takahashi, ${ }^{7}$ A. H. Tang, ${ }^{3}$ Z. Tang, ${ }^{38}$ L. H. Tarini,${ }^{50}$ T. Tarnowsky, ${ }^{24}$ D. Thein ${ }^{43}$ J. H. Thomas, ${ }^{21}$ J. Tian, ${ }^{40}$ A. R. Timmins ${ }^{50}$ S. Timoshenko, ${ }^{25}$ D. Tlusty, ${ }^{11}$ M. Tokarev,${ }^{17}$ T. A. Trainor, ${ }^{49}$ V. N. Tram,${ }^{21}$ S. Trentalange, ${ }^{6}$ R. E. Tribble,${ }^{42}$ O. D. Tsai, ${ }^{6}$ J. Ulery, ${ }^{33}$ T. Ullrich, ${ }^{3}$ D. G. Underwood, ${ }^{1}$ G. Van Buren, ${ }^{3}$ G. van Nieuwenhuizen, ${ }^{22}$ J. A. Vanfossen Jr., ${ }^{18}$ R. Varma, ${ }^{13}$ G. M. S. Vasconcelos, ${ }^{7}$ A. N. Vasiliev, ${ }^{32}$ F. Videbaek, ${ }^{3}$ S. E. Vigdor,${ }^{14}$ Y. P. Viyogi,,${ }^{12}$ S. Vokal, ${ }^{17}$ S. A. Voloshin, ${ }^{50}$ M. Wada, ${ }^{43}$ M. Walker, ${ }^{22}$ F. Wang, ${ }^{33}$ G. Wang, ${ }^{6}$ H. Wang, ${ }^{24}$ J. S. Wang, ${ }^{20}$ Q. Wang, ${ }^{33}$ X. Wang,${ }^{44}$ X. L. Wang, ${ }^{38}$ Y. Wang, ${ }^{44}$ G. Webb, ${ }^{19}$

J. C. Webb, ${ }^{46}$ G. D. Westfall, ${ }^{24}$ C. Whitten Jr. ${ }^{6}$ H. Wieman, ${ }^{21}$ S. W. Wissink,${ }^{14}$ R. Witt ${ }^{45} \mathrm{Y} . \mathrm{Wu},{ }^{51}$ W. Xie, ${ }^{33}$ N. Xu, ${ }^{21}$

Q. H. Xu, ${ }^{39}$ Y. Xu,${ }^{38}$ Z. Xu, ${ }^{3}$ Y. Yang, ${ }^{20}$ P. Yepes ${ }^{36}$ K. Yip, ${ }^{3}$ I.-K. Yoo, ${ }^{34}$ Q. Yue, ${ }^{44}$ M. Zawisza, ${ }^{48}$ H. Zbroszczyk, ${ }^{48}$ W. Zhan, ${ }^{20}$ S. Zhang, ${ }^{40}$ W. M. Zhang, ${ }^{18}$ X. P. Zhang, ${ }^{21}$ Y. Zhang, ${ }^{21}$ Z. P. Zhang, ${ }^{38}$ Y. Zhao, ${ }^{38}$ C. Zhong, ${ }^{40}$ J. Zhou, ${ }^{36}$ X. Zhu, ${ }^{44}$

R. Zoulkarneev, ${ }^{17}$ Y. Zoulkarneeva, ${ }^{17}$ and J. X. Zuo ${ }^{40}$

(STAR Collaboration)

${ }^{1}$ Argonne National Laboratory, Argonne, Illinois 60439, USA

${ }^{2}$ University of Birmingham, Birmingham, United Kingdom

${ }^{3}$ Brookhaven National Laboratory, Upton, New York 11973, USA

${ }^{4}$ University of California, Berkeley, California 94720, USA

${ }^{5}$ University of California, Davis, California 95616, USA

${ }^{6}$ University of California, Los Angeles, California 90095, USA

${ }^{7}$ Universidade Estadual de Campinas, Sao Paulo, Brazil

${ }^{8}$ University of Illinois at Chicago, Chicago, Illinois 60607, USA

${ }^{9}$ Creighton University, Omaha, Nebraska 68178, USA 
${ }^{10}$ Czech Technical University in Prague, Faculty of Nuclear Sciences and Physical Engineering, CZ-115 19 Prague, Czech Republic

${ }^{11}$ Nuclear Physics Institute of the Academy of Sciences of the Czech Republic, CZ-250 $68 \check{R}$ ě̃/Prague, Czech Republic

${ }^{12}$ Institute of Physics, Bhubaneswar 751005, India

${ }^{13}$ Indian Institute of Technology, Mumbai, India

${ }^{14}$ Indiana University, Bloomington, Indiana 47408, USA

${ }^{15}$ Institut de Recherches Subatomiques, Strasbourg, France

${ }^{16}$ University of Jammu, Jammu 180001, India

${ }^{17}$ Joint Institute for Nuclear Research, Dubna, RU-141 980, Russia

${ }^{18}$ Kent State University, Kent, Ohio 44242, USA

${ }^{19}$ University of Kentucky, Lexington, Kentucky, 40506-0055, USA

${ }^{20}$ Institute of Modern Physics, Lanzhou, People's Republic of China

${ }^{21}$ Lawrence Berkeley National Laboratory, Berkeley, California 94720, USA

${ }^{22}$ Massachusetts Institute of Technology, Cambridge, Massachusetts 02139-4307, USA

${ }^{23}$ Max-Planck-Institut für Physik, Munich, Germany

${ }^{24}$ Michigan State University, East Lansing, Michigan 48824, USA

${ }^{25}$ Moscow Engineering Physics Institute, Moscow, Russia

${ }^{26}$ City College of New York, New York City, New York 10031, USA

${ }^{27}$ NIKHEF and Utrecht University, Amsterdam, The Netherlands

${ }^{28}$ Ohio State University, Columbus, Ohio 43210, USA

${ }^{29}$ Old Dominion University, Norfolk, Virginia 23529, USA

${ }^{30}$ Panjab University, Chandigarh 160014, India

${ }^{31}$ Pennsylvania State University, University Park, Pennsylvania 16802, USA

${ }^{32}$ Institute of High Energy Physics, Protvino, Russia

${ }^{33}$ Purdue University, West Lafayette, Indiana 47907, USA

${ }^{34}$ Pusan National University, Pusan, Republic of Korea

${ }^{35}$ University of Rajasthan, Jaipur 302004, India

${ }^{36}$ Rice University, Houston, Texas 77251, USA

${ }^{37}$ Universidade de Sao Paulo, Sao Paulo, Brazil

${ }^{38}$ University of Science \& Technology of China, Hefei 230026, People's Republic of China

${ }^{39}$ Shandong University, Jinan, Shandong 250100, People's Republic of China

${ }^{40}$ Shanghai Institute of Applied Physics, Shanghai 201800, People's Republic of China

${ }^{41}$ SUBATECH, Nantes, France

${ }^{42}$ Texas A\&M University, College Station, Texas 77843, USA

${ }^{43}$ University of Texas, Austin, Texas 78712, USA

${ }^{44}$ Tsinghua University, Beijing 100084, People's Republic of China

${ }^{45}$ United States Naval Academy, Annapolis, Maryland 21402, USA

${ }^{46}$ Valparaiso University, Valparaiso, Indiana 46383, USA

${ }^{47}$ Variable Energy Cyclotron Centre, Kolkata 700064, India

${ }^{48}$ Warsaw University of Technology, Warsaw, Poland

${ }^{49}$ University of Washington, Seattle, Washington 98195, USA

${ }^{50}$ Wayne State University, Detroit, Michigan 48201, USA

${ }^{51}$ Institute of Particle Physics, CCNU (HZNU), Wuhan 430079, People's Republic of China

${ }^{52}$ Yale University, New Haven, Connecticut 06520, USA

${ }^{53}$ University of Zagreb, Zagreb HR-10002, Croatia

(Received 1 September 2009; published 29 December 2009)

The STAR Collaboration at the Relativistic Heavy Ion Collider presents a systematic study of high-transversemomentum charged-di-hadron correlations at small azimuthal pair separation $\Delta \phi$ in $d+\mathrm{Au}$ and central $\mathrm{Au}+\mathrm{Au}$ collisions at $\sqrt{s_{N N}}=200 \mathrm{GeV}$. Significant correlated yield for pairs with large longitudinal separation $\Delta \eta$ is observed in central $\mathrm{Au}+\mathrm{Au}$ collisions, in contrast to $d+\mathrm{Au}$ collisions. The associated yield distribution in $\Delta \eta \times \Delta \phi$ can be decomposed into a narrow jet-like peak at small angular separation which has a similar shape to that found in $d+$ Au collisions, and a component that is narrow in $\Delta \phi$ and depends only weakly on $\Delta \eta$, the "ridge." Using two systematically independent determinations of the background normalization and shape, finite ridge yield is found to persist for trigger $p_{t}>6 \mathrm{GeV} / c$, indicating that it is correlated with jet production. The transverse-momentum spectrum of hadrons comprising the ridge is found to be similar to that of bulk particle production in the measured range $\left(2<p_{t}<4 \mathrm{GeV} / c\right)$. 


\section{INTRODUCTION}

Measurements of inclusive hadron suppression [1,2] and di-hadron azimuthal correlations [3-5] in ultrarelativistic nuclear collisions have provided important insights into the properties of hot quantum chromodynamic (QCD) matter [6-9]. In particular, the high-transverse-momentum (high- $p_{t}$ ) suppression [5] and low- $p_{t}$ enhancement [4] of the correlated yield of hadrons recoiling from a high- $p_{t}$ particle (azimuthal pair separation $\Delta \phi \sim \pi$ ) suggest a dramatic softening of jet fragmentation in dense matter, arising from strong partonic energy loss.

Studies of near-side (small- $\Delta \phi$ ) di-hadron correlations in events containing a "trigger particle" at high $p_{t}(>6$ $\mathrm{GeV} / c$ ) reveal a jet-like correlation at small angular separation (small pseudorapidity pair separation $\Delta \eta$ and small $\Delta \phi$ ), which is unmodified in central $\mathrm{Au}+\mathrm{Au}$ collisions relative to $d+\mathrm{Au}$ collisions [5], suggesting that the dominant production mechanism is jet fragmentation outside the dense medium.

At lower trigger momentum, significant near-side correlated yield has been observed at large pair separation ( $\Delta \eta \sim 1$ ) [4], whereas, for untriggered correlations, longitudinal broadening at small $\Delta \eta$ has been seen $[10,11]$. However, inclusive hadron production at moderate $p_{t}<$ $6 \mathrm{GeV} / c$ in central $\mathrm{Au}+\mathrm{Au}$ collisions differs significantly from that observed in more elementary collision systems $[12,13]$, indicating that jet fragmentation may not be the dominant hadron production mechanism in the kinematic region of these studies. For example, the large baryon-tomeson ratio observed at intermediate $p_{t}$ is generally attributed to hadron formation by coalescence of constituent quarks $[14,15]$, which might also affect the di-hadron correlation structure [16].

This article presents new measurements using the STAR detector to explore the near-side correlation structure in $\mathrm{Au}+\mathrm{Au}$ and $d+\mathrm{Au}$ collisions at $\sqrt{s_{N N}}=200 \mathrm{GeV}$, with emphasis on the $\Delta \eta$ shape and high- $p_{t}$ trigger particles. In central $\mathrm{Au}+\mathrm{Au}$ collisions, a significant associated yield at large $\Delta \eta$ is observed for all $p_{t}^{\text {trig }}$, including $p_{t}^{\text {trig }}>6 \mathrm{GeV} / c$, where jet fragmentation is expected to be the dominant particle production mechanism. At large $p_{t}^{\text {trig }}$ the near-side correlation structure can be factored into a jet-like peak, with properties similar to correlations in $p+p$ collisions, and an elongated contribution that is approximately independent of $\Delta \eta$, which we therefore call the ridge. Similar phenomena have been observed for lower $p_{t}^{\text {trig }}$ and $p_{t}^{\text {assoc }}$ over a broader interval in $\Delta \eta[17]$.

Based on earlier measurements and preliminary versions of some of the results in the present article, several models have been proposed to explain the observed broadening of the near-side distributions and the occurrence of the ridge. Models based on radiative partonic energy loss suggest that the ridge arises from the coupling of induced gluon radiation with the longitudinal flow of bulk matter [18], or from the coupling of radiation to transverse chromomagnetic fields [19,20]. Other models attribute the ridge to the effect of elastic scattering of the jet in the flowing medium [21], to medium heating by a jet [22], to radial flow of bulk matter in coincidence with a jet trigger bias due to energy loss [23,24], or to long-range rapidity correlations arising from a color glass condensate (CGC) initial state $[25,26]$.

To address some of the model expectations, we study not only the shape of di-hadron correlations in $\Delta \eta$ and $\Delta \phi$ but also the $p_{t}$ dependence of the correlated yield. The ridge yield at high $p_{t}^{\text {trig }}$ is examined using two systematically independent assessments of the background contribution of uncorrelated tracks. Comparison is made to $d+$ Au reference data to quantify the modification of jet fragmentation due to interactions in the hot medium.

\section{EXPERIMENTAL SETUP}

The measurements were carried out by the STAR Collaboration at the Relativistic Heavy Ion Collider (RHIC) [27] using 12 million minimum bias $d+\mathrm{Au}$ events from RHIC Run 3, and 13 million central $\mathrm{Au}+\mathrm{Au}$ collisions at $\sqrt{s_{N N}}=200 \mathrm{GeV}$ from RHIC Run 4 after event cuts. Triggering, centrality selection, and tracking used standard STAR procedures [1,5,28]. Primary-vertex tracks within $|\eta|<1$ were selected for this analysis using standard quality cuts on the number of hits in the Time Projection Chamber (TPC) and the distance of closest approach to the primary vertex, which eliminate fake tracks and ensure sufficient momentum resolution for high- $p_{t}$ measurements [1,28]. The effect of track merging is negligible in the kinematic range used in this analysis.

The central $\mathrm{Au}+\mathrm{Au}$ events used in this analysis were selected during data gathering based on signals in the zerodegree calorimeters (ZDCs) [27] and a cut on the multiplicity in the central trigger barrel to reject peripheral events. The trigger selected the most central $12 \%$ of the total hadronic cross section, which we label "central $\mathrm{Au}+\mathrm{Au}$ " in the following. The $d+$ Au events used in this analysis were selected using a minimum bias trigger requiring at least one beam-rapidity neutron in the ZDC in the $\mathrm{Au}$ beam direction (negative pseudorapidity) accepting $95 \pm 3 \%$ of the $d+\mathrm{Au}$ hadronic cross section [28].

\section{III. $\Delta \eta \times \Delta \phi$ DI-HADRON CORRELATIONS}

The event-averaged associated hadron distribution, formed using pairs of charged primary tracks within certain $p_{t}$ intervals for the trigger and associated particles, is calculated as

$$
\frac{d^{2} N}{d \Delta \phi d \Delta \eta}(\Delta \phi, \Delta \eta)=\frac{1}{N_{\text {trig }}} \frac{1}{\epsilon(\phi, \eta, \Delta \phi, \Delta \eta)} \frac{d^{2} N_{\text {raw }}}{d \Delta \phi d \Delta \eta},
$$

where $\Delta \phi$ and $\Delta \eta$ are the azimuthal and pseudorapidity separation of the pair, $N_{\text {trig }}$ is the number of trigger particles, and $d^{2} N_{\text {raw }} / d \Delta \phi d \Delta \eta$ is the measured di-hadron distribution. The factor $1 / \epsilon(\phi, \eta, \Delta \phi, \Delta \eta)$ accounts for the reconstruction efficiency of associated tracks, determined by embedding simulated single tracks into real events, and for the limited acceptance in $\eta$ and TPC sector boundaries in $\phi$, determined 

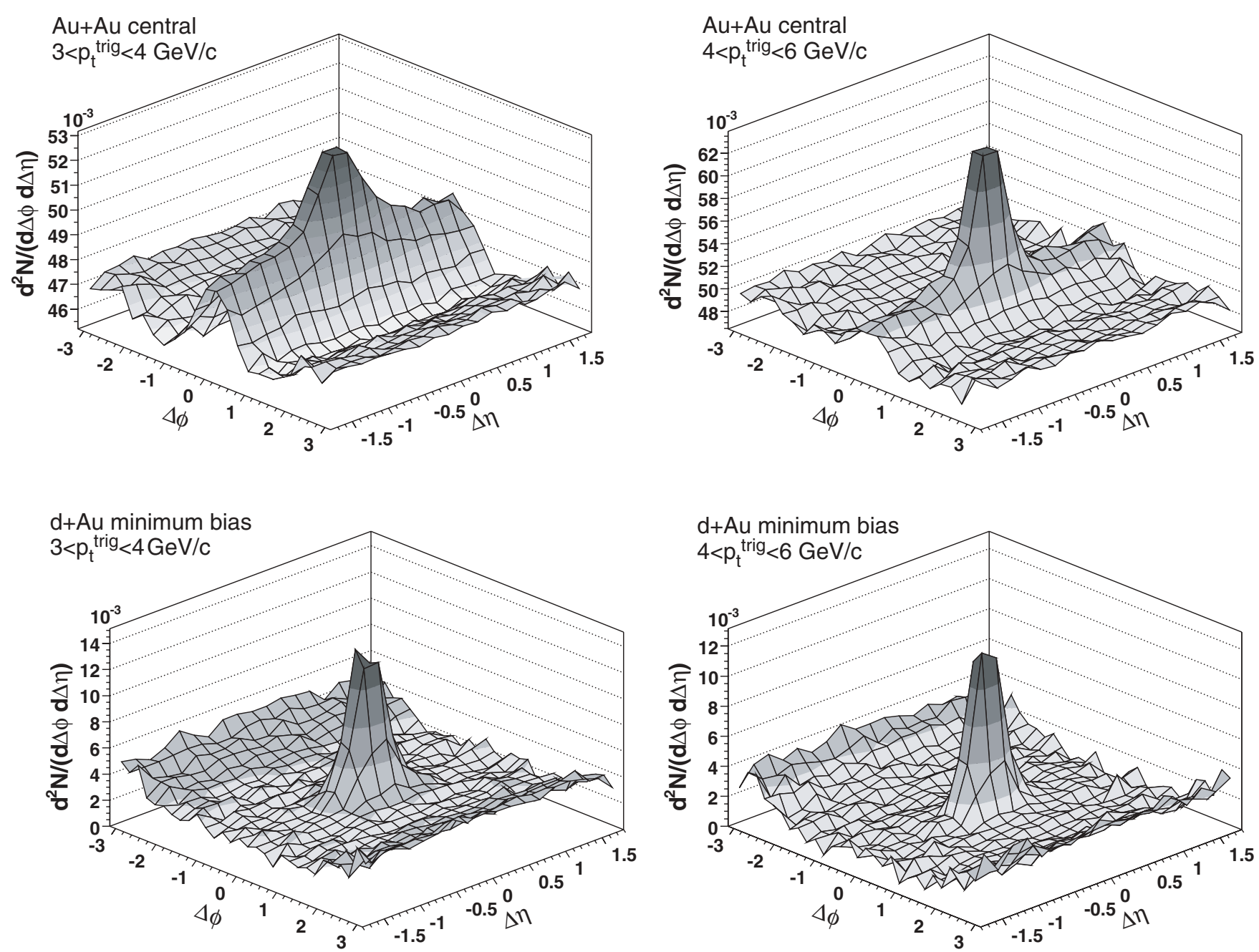

FIG. 1. Charged-di-hadron distribution [Eq. (1)] for $2 \mathrm{GeV} / c<p_{t}^{\text {assoc }}<p_{t}^{\text {assoc }}$. Upper left: central Au $+\mathrm{Au}, 3<p_{t}^{\text {trig }}<4 \mathrm{GeV} / c$; upper right: central $\mathrm{Au}+\mathrm{Au}, 4<p_{t}^{\text {trig }}<6 \mathrm{GeV} / c$; lower left: minimum bias $d+\mathrm{Au}, 3<p_{t}^{\text {trig }}<4 \mathrm{GeV} / c$; lower right: minimum bias $d+\mathrm{Au}$, $4<p_{t}^{\text {trig }}<6 \mathrm{GeV} / c$. Note the different vertical scales, as well as the suppressed zero in the upper panels.

by event mixing. Associated particles have $2<p_{t}^{\text {assoc }}<$ $p_{t}^{\text {trig }} \mathrm{GeV} / c$ for consistency with previous results [5], except for a new analysis, which directly compares correlations for different $p_{t}^{\text {trig }}$ (Section VIA), where $2<p_{t}^{\text {assoc }}<4 \mathrm{GeV} / c$ was used.

Figure 1 shows distributions of the associated particle yield defined in Eq. (1) for central $\mathrm{Au}+\mathrm{Au}$ events with triggers $3<p_{t}^{\text {trig }}<4$ and $4<p_{t}^{\text {trig }}<6 \mathrm{GeV} / c$ (upper panels) and for $d+$ Au events with the same $p_{t}^{\text {trig }}$ selections (lower panels). A near-side peak centered on $(\Delta \eta, \Delta \phi)=(0,0)$ is evident in all panels and is consistent with jet fragmentation. In addition, a significant enhancement of near-side correlated yield is seen at large $\Delta \eta$ for central $\mathrm{Au}+\mathrm{Au}$ events but not for $d+\mathrm{Au}$ events: the ridge.

In this analysis we examine the shape of the near-side associated yield distribution in detail via projections on the $\Delta \eta$ and $\Delta \phi$ axes. We characterize the shapes of both the ridge and the jet-like peak and study the $p_{t}$ dependence of the ridge and jet-like yields.

\section{RIDGE SHAPE IN $\Delta \eta$}

To study the ridge quantitatively, the di-hadron distribution is projected onto the $\Delta \eta$ axis in intervals of $\Delta \phi$ :

$$
\left.\frac{d N}{d \Delta \eta}\right|_{a, b} \equiv \int_{a}^{b} d \Delta \phi \frac{d^{2} N}{d \Delta \phi d \Delta \eta}
$$

similarly for projection onto $\Delta \phi$ :

$$
\left.\frac{d N}{d \Delta \phi}\right|_{a, b} \equiv \int_{|\Delta \eta| \in[a, b]} d \Delta \eta \frac{d^{2} N}{d \Delta \phi d \Delta \eta} .
$$

The contribution to the di-hadron distribution of elliptic flow $\left(v_{2}\right)$ in nuclear collisions [3] is estimated via

$$
B_{\Delta \phi}[a, b] \equiv b_{\Delta \phi} \int_{a}^{b} d \Delta \phi\left(1+2\left\langle v_{2}^{\text {trig }} v_{2}^{\text {assoc }}\right\rangle \cos 2 \Delta \phi\right),
$$

where the mean uncorrelated level $b_{\Delta \phi}$ is fixed by the assumption of zero correlated yield at the minimum of the projected distribution, in this case $1.0<\Delta \phi<1.2$ (zero 
TABLE I. Values of $b_{\Delta \phi}$ and $b_{\Delta \eta}$ used for the ZYAM normalization in Eqs. (4) and (6) for different $p_{t}^{\text {trig }}$ windows, with $2 \mathrm{GeV} / c<p_{t}^{\text {assoc }}<p_{t}^{\text {trig }}$. Errors are statistical only.

\begin{tabular}{lcc}
\hline \hline$p_{t}^{\text {trig }}(\mathrm{GeV} / c)$ & $b_{\Delta \phi}$ & $b_{\Delta \eta}$ \\
\hline $3-4$ & $0.4302 \pm\left(1 \times 10^{-4}\right)$ & $0.1245 \pm\left(1 \times 10^{-4}\right)$ \\
$4-5$ & $0.4502 \pm\left(4 \times 10^{-4}\right)$ & $0.1296 \pm\left(3 \times 10^{-4}\right)$ \\
$5-6$ & $0.4533 \pm\left(8 \times 10^{-4}\right)$ & $0.1295 \pm\left(6 \times 10^{-4}\right)$ \\
$6-10$ & $0.4508 \pm\left(1 \times 10^{-3}\right)$ & $0.1284 \pm\left(1 \times 10^{-3}\right)$ \\
\hline \hline
\end{tabular}

yield at minimum, or ZYAM) $[3,4,29,30]$. Values of $b_{\Delta \phi}$ are given in Table I. The modulation amplitude $\left\langle v_{2}^{\text {trig }} v_{2}^{\text {assoc }}\right\rangle$ is approximated as $\left\langle v_{2}^{\text {trig }}\right\rangle\left\langle v_{2}^{\text {assoc }}\right\rangle$ using the mean of the event plane, determined at forward rapidities in the Forward TPCs [FTPCs; $\left.v_{2}\{\mathrm{FTPC}\}\left(p_{t}\right)\right]$ and four-particle cumulant methods $\left[v_{2}\{4\}\left(p_{t}\right)\right]$ [31] (see Table II). In general, the correlation structures seen in Fig. 1 also affect the $v_{2}$ measurement. In particular the near-side ridge structure may bias the event-plane determination. This effect is reduced by using forward-rapidity tracks for the event-plane determination in the $v_{2}\{\mathrm{FTPC}\}$ method and by using four-particle correlations in the $v_{2}\{4\}$ method. The $v_{2}\{4\}$ method is also sensitive to $v_{2}$ fluctuations, which reduce the measured value, and is therefore used as a lower systematic limit. The $v_{2}$ systematic uncertainty is defined using $v_{2}\{$ FTPC $\}$ as a maximum and $v_{2}\{4\}$ as a minimum in each $p_{t}$ bin. An alternative to the ZYAM procedure is discussed in Section VI A.

The near-side correlated yield within a $\Delta \eta$ interval of width $\delta$ is then

$$
\begin{aligned}
Y_{\text {slice }}(\Delta \eta ; \delta)= & \int_{-0.7}^{0.7} d \Delta \phi\left(\left.\frac{d N}{d \Delta \phi}\right|_{\Delta \eta-\delta / 2, \Delta \eta+\delta / 2}\right) \\
& -B_{\Delta \phi}[-0.7,0.7] .
\end{aligned}
$$

The systematic uncertainty on $Y_{\text {slice }}(\Delta \eta ; \delta)$ includes contributions from $v_{2}$ but not from the ZYAM assumption. The statistical error of $b_{\Delta \phi}$, which is determined independently for every $\Delta \eta$ interval using the ZYAM procedure [and $b_{\Delta \eta}$ in Eq. (6)] is included in the error on $Y_{\text {slice }}(\Delta \eta ; \delta)$ [and $Y_{\text {ridge }}$ in Eq. (9)].

Figure 2 shows $Y_{\text {slice }}(\Delta \eta ; \delta=0.3)$ as a function of $\Delta \eta$; $Y_{\text {slice }}$ is largest around $\Delta \eta=0$, as expected from jet fragmentation. However, a significant associated yield is also seen at large $\Delta \eta>1$, for all $p_{t}^{\text {trig }}$. The systematic uncertainties in

TABLE II. Elliptic flow $\left(v_{2}\right)$ values for different $p_{t}^{\text {trig }}$ and $p_{t}^{\text {assoc }}$ windows, defined as the mean of the FTPC reaction plane $\left[v_{2}\{\operatorname{FTPC}\}\left(p_{t}\right)\right]$ and four-particle cumulant methods $\left[v_{2}\{4\}\left(p_{t}\right)\right]$ in central $\mathrm{Au}+\mathrm{Au}$ collisions $\left(v_{2}(\mathrm{Mean})\right)$. Uncertainties are the variation in $v_{2}$ from these two approaches.

\begin{tabular}{lccc}
\hline \hline$p_{t}^{\text {trig }}(\mathrm{GeV} / c)$ & $v_{2}($ Mean $)(\%)$ & $p_{t}^{\text {assoc }}(\mathrm{GeV} / c)$ & $v_{2}$ (Mean) $(\%)$ \\
\hline $3-4$ & $8.5 \pm 2.2$ & $2.0-2.5$ & $8.0 \pm 1.7$ \\
$4-5$ & $7.7 \pm 1.9$ & $2.5-3.0$ & $8.4 \pm 1.9$ \\
$5-6$ & $6.5 \pm 1.5$ & $3.0-3.5$ & $8.5 \pm 2.1$ \\
$6-10$ & $4.6 \pm 1.3$ & $3.5-4.0$ & $8.3 \pm 2.1$ \\
\hline \hline
\end{tabular}

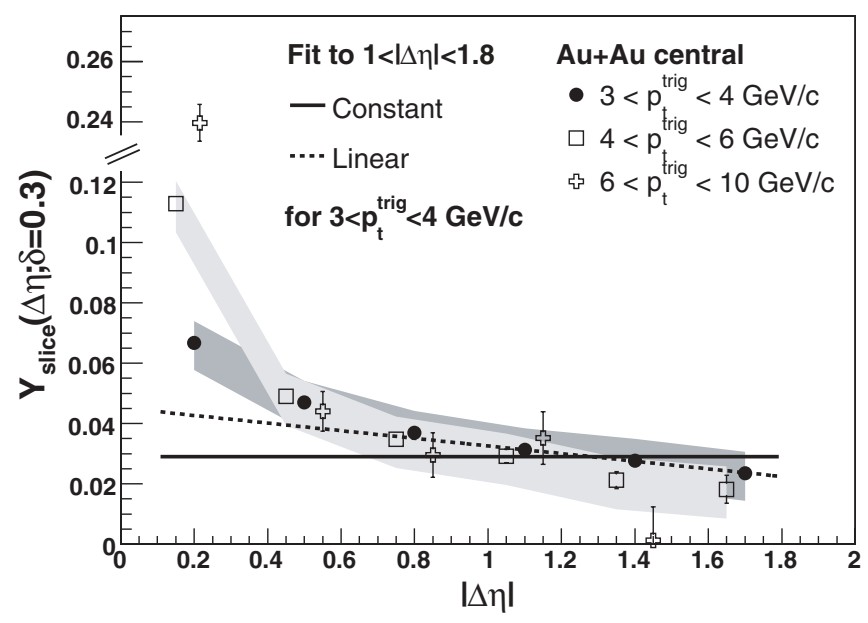

FIG. 2. $Y_{\text {slice }}(\Delta \eta ; \delta=0.3)$ [Eq. (5)] for central $\mathrm{Au}+\mathrm{Au}$ collisions, $2 \mathrm{GeV} / c<p_{t}^{\text {assoc }}<p_{t}^{\text {trig }}$, and various $p_{t}^{\text {trig }}$ vs $\Delta \eta$; the shaded bands represents the systematic uncertainties due to $v_{2}$ (not shown for $\left.6<p_{t}^{\text {trig }}<10 \mathrm{GeV} / c\right)$. The solid and dashed lines represent constant or linear fit to $1<|\Delta \eta|<1.8$; only shown for $3<p_{t}^{\text {trig }}<4 \mathrm{GeV} / c$ (see text). Some data points are displaced horizontally for clarity.

the figure are due to the uncertainty of the elliptic flow of the background, which may be $\Delta \eta$ dependent. The yield at large $\Delta \eta$ exhibits no significant dependence on $\Delta \eta$ within the experimental acceptance and the statistical and systematic uncertainties.

A fit to the three data points at largest $|\Delta \eta|$ in Fig. 2 for the $p_{t}^{\text {trig }}$ intervals $3-4,4-6$, and $6-10 \mathrm{GeV} / c$ was used to estimate the total ridge yield using the two assumptions of no $\Delta \eta$ dependence or linear ridge variation with $\Delta \eta$. These two cases delimit the unknown ridge yield at small $\Delta \eta$. For all $p_{t}^{\text {trig }}$ bins, the assumption of linear variation gives an estimated total ridge yield that is $10-15 \%$ larger than the assumption of a $\Delta \eta$-independent ridge. The following discussion assumes that the ridge is independent on $\Delta \eta$, but the systematic uncertainty assigned to the ridge yield includes the linear-variation case.

\section{CHARACTERIZATION OF JET-LIKE PEAK}

Based on these observations, we separate the near-side projection onto the $\Delta \eta$ axis in a jet-like peak centered at $\Delta \eta=0$ and a $\Delta \eta$-independent ridge component. Because $v_{2}$ measured within the acceptance of this analysis has negligible variation with $\eta$ [31], the jet-like yield may be written as

$$
\frac{d N_{J}}{d \Delta \eta}(\Delta \eta)=\left.\frac{d N}{d \Delta \eta}\right|_{-0.7,0.7}-b_{\Delta \eta},
$$

where the constant background level $b_{\Delta \eta}$ is calculated in the interval $1.0<|\Delta \eta|<1.7$. Values of $b_{\Delta \eta}$ are given in Table I.

The jet-like yield can alternatively be defined by projecting onto the $\Delta \phi$ axis, assuming negligible jet-like contribution in $|\Delta \eta|>0.7$ :

$$
\frac{d N_{J}}{d \Delta \phi}(\Delta \phi)=\left.\frac{d N}{d \Delta \phi}\right|_{0,0.7}-\left.\frac{d N}{d \Delta \phi}\right|_{0.7,1.4} .
$$



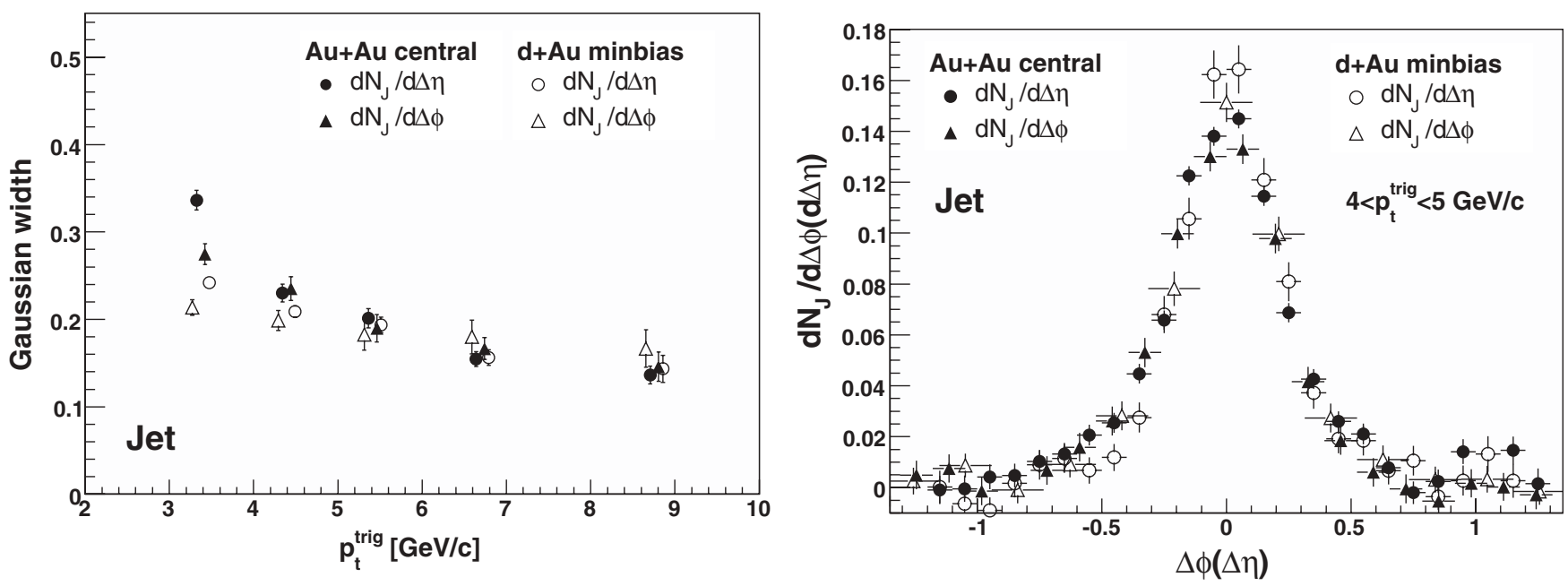

FIG. 3. Left panel: width of Gaussian fit to jet-like peak for Eq. (6) ( $\Delta \eta$ width, circles) and Eq. (7) $(\Delta \phi$ width, triangles); 2 GeV/c $<$ $p_{t}^{\text {assoc }}<p_{t}^{\text {trig }}$, as a function of $p_{t}^{\text {trig }}$, for central $\mathrm{Au}+\mathrm{Au}$ collisions (filled symbols) and $d+\mathrm{Au}$ collisions (open symbols). Some data points are displaced horizontally for clarity. Right panel: the distributions of Eqs. (6) and (7) for $4<p_{t}^{\text {trig }}<5 \mathrm{GeV} / c$ and $2 \mathrm{GeV} / c<p_{t}^{\text {assoc }}<p_{t}^{\text {trig }}$.

The left-hand panel of Fig. 3 shows the widths of Gaussian fits to $d N_{J} / d \Delta \eta$ and $d N_{J} / d \Delta \phi$ versus $p_{t}^{\text {trig }}$, for both central $\mathrm{Au}+\mathrm{Au}$ and $d+\mathrm{Au}$ events. At low $p_{t}^{\text {trig }}$ the jet-like peak is significantly broadened in $\mathrm{Au}+\mathrm{Au}$ relative to $d+\mathrm{Au}$. Similar broadening has been observed previously at low $p_{t}^{\text {trig }}[4,10]$.

For $p_{t}^{\text {trig }}>5 \mathrm{GeV} / c$, the jet-like peak has similar width in $\Delta \eta$ and $\Delta \phi$ that is consistent with $d+$ Au reference measurements. The full distributions for the two projections are shown in the right-hand panel of Fig. 3 for central $\mathrm{Au}+\mathrm{Au}$ and $d+\mathrm{Au}$ minimum bias, for $4<p_{t}^{\text {trig }}<5$ and $p_{t}^{\text {assoc }}>2 \mathrm{GeV} / c$. The similarity suggests that, for high $p_{t}^{\text {trig }}$, the near-side jet-like peak arises from jet fragmentation in a vacuum, with little modification by the medium for $p_{t}^{\text {assoc }}>$ $2 \mathrm{GeV} / c$. Note that this observation does not preclude significant jet energy loss prior to fragmentation of the leading parton.

\section{CHARACTERIZATION OF THE RIDGE, $p_{t}^{\text {trig }}$ DEPENDENCE}

The yield of the jet-like peak $Y_{J}^{\Delta \eta}$ and the yield of the ridge $Y_{\text {ridge }}$ are obtained by suitable integrals over Eqs. (5) and (6):

$$
\begin{aligned}
Y_{J}^{\Delta \eta} & =\int_{-0.7}^{0.7} d \Delta \eta \frac{d N_{J}}{d \Delta \eta}(\Delta \eta), \\
Y_{\text {ridge }} & =\left.\int_{-1.7}^{1.7} d \Delta \eta \frac{d N}{d \Delta \eta}\right|_{-0.7,0.7}-B_{\Delta \phi}[-0.7,0.7]-Y_{J}^{\Delta \eta} .
\end{aligned}
$$

Figure 4 shows $Y_{\text {ridge }}$ as function of $p_{t}^{\text {trig }}$ for central $\mathrm{Au}+$ Au collisions, using ZYAM to normalize the background level. Significant ridge yield is observed for all $p_{t}^{\text {trig }}$, in particular for $p_{t}^{\text {trig }}>6 \mathrm{GeV} / c$, where jet fragmentation is expected to be the dominant hadron production mechanism even in nuclear collisions $[12,13]$.

\section{A. Independent estimate of lower bound on ridge yield}

The preceding conclusion relies on both the two-component model of jet and background and the ZYAM background normalization assumption and $v_{2}$ correction for background. However, ZYAM does not provide a strict lower or upper bound on the combinatorial di-hadron background. An estimate of the combinatorial background that is systematically independent of these assumptions can be obtained by attributing the recoil yield entirely to elliptic flow and comparing the near-side yield (small $\Delta \phi$ ) to the recoil yield in the ridge region $|\Delta \eta|>0.7$.

Because finite jet-correlated recoil yield has been observed over background for $p_{t}^{\text {trig }}>6 \mathrm{GeV} / c$ and $p_{t}^{\text {assoc }}>2 \mathrm{GeV} / c$ [5], this procedure overestimates the near-side combinatorial background and therefore underestimates the extracted ridge yield. Full correction for this effect requires theoretical modeling that is beyond the scope of the present work. To minimize

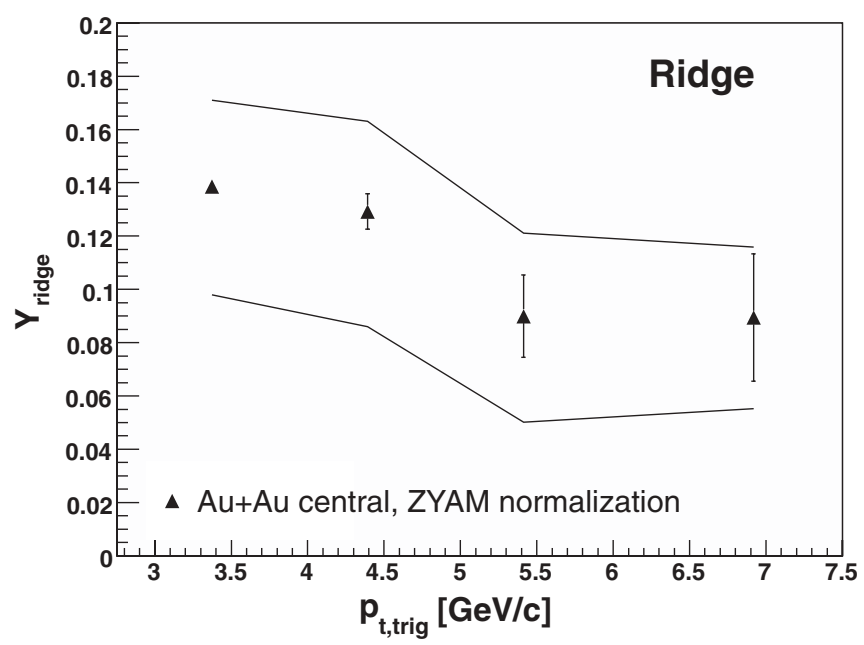

FIG. 4. Ridge yield [Eq. (9)] in $|\Delta \eta|<1.7$ and $2 \mathrm{GeV} / c<$ $p_{t}^{\text {assoc }}<p_{t}^{\text {trig }}$ as a function of $p_{t}^{\text {trig }}$. Solid lines are the systematic uncertainty due to $v_{2}$. 


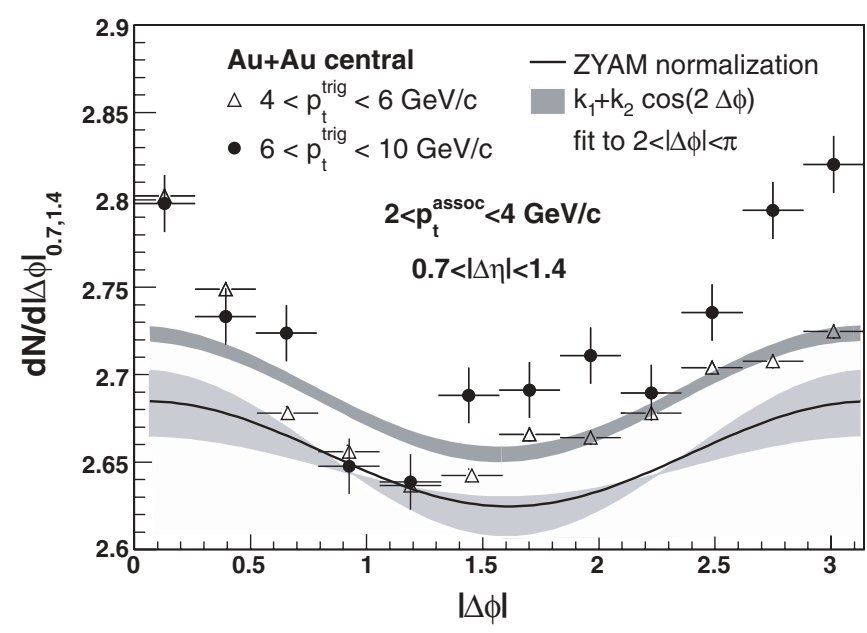

FIG. 5. Projection of $d N /\left.d \Delta \phi\right|_{a, b}$ for $0.7<|\Delta \eta|<1.4$ [Eq. (3)] in two trigger $p_{t}$ windows for $2<p_{t}^{\text {assoc }}<4 \mathrm{GeV} / c$. No background subtraction has been applied; note the suppressed zero on the vertical scale. The shaded band shows the fit of the function $k_{1}+k_{2}$. $\cos (2 \Delta \phi)$ to the recoil region $2<|\Delta \phi|<\pi$ for $4<p_{t}^{\text {trig }}<6 \mathrm{GeV} / c$. The width of the band indicates the fitting error. The solid curve represents the background estimate using the ZYAM normalization for $4<p_{t}^{\text {trig }}<6 \mathrm{GeV} / c$. Systematic uncertainties are indicated by the light shaded band.

this effect we use the observation that the combinatorial background level is dominantly a function only of $p_{t}^{\text {assoc }}$ [5] and we estimate the maximum background yield for all $p_{t}^{\text {trig }}$ using the recoil distribution for $4<p_{t}^{\text {trig }}<6 \mathrm{GeV} / c$, where the jet-correlated recoil yield in central events is small compared to the background for $p_{t}^{\text {assoc }}>2 \mathrm{GeV} / c$. A possible multiplicity bias due to the presence of a high- $p_{t}$ trigger particle is estimated to be around $0.1 \%$. This bias provides a significant lower bound to the ridge yield.

Figure 5 shows $d N /\left.d \Delta \phi\right|_{0.7,1.4}$ [Eq. (3)] for $6<p_{t}^{\text {trig }}<$ $10 \mathrm{GeV} / c$ (solid circles) and $4<p_{t}^{\text {trig }}<6 \mathrm{GeV} / c$ (triangles), for $2<p_{t}^{\text {assoc }}<4 \mathrm{GeV} / c$. No background correction has been applied; note the suppressed zero on the vertical axis. Error bars are statistical. The systematic uncertainty of associated hadron yields is dominated by a $5 \%$ uncertainty in the tracking efficiency $\epsilon(\Delta \phi, \Delta \eta)$ in Eq. (1). The high- $p_{t}$ tracks used for these distributions are long, relatively straight tracks with similar topology; therefore, the uncorrelated systematic uncertainty of the distributions in Fig. 5 is negligible relative to the statistical errors.

The shaded band in the figure shows the fit of the function $k_{1}+k_{2} \cdot \cos (2 \Delta \phi)$ to the recoil distribution in the region $2<$ $|\Delta \phi|<\pi$ for $4<p_{t}^{\text {trig }}<6 \mathrm{GeV} / c$. A small but significant excess is seen at $|\Delta \phi|<0.5$ for the signal relative to the band for both $4<p_{t}^{\text {trig }}<6 \mathrm{GeV} / c$ and $6<p_{t}^{\text {trig }}<10 \mathrm{GeV} / c$, corresponding to the ridge yield. The measured distributions undershoot this background estimate in the region $0.5<$ $\Delta \phi<1.5$, which may indicate that this method overestimates the background somewhat because of the presence of a small recoil yield even for the lower $p_{t}^{\text {trig }}$ selection. However, it is also possible that the presence of a trigger locally depletes the correlated yield relative to an uncorrelated background, in which case the ZYAM procedure would underestimate the background and the alternative procedure would be more appropriate.

The solid line with the light shaded band around it in Fig. 5 indicates the combinatorial background estimation using the ZYAM assumption. By construction, this assumption does not admit an undershoot of the measured distribution relative to the background. Larger ridge yield is estimated using this technique. Note that these estimates do not provide a strict lower bound on the background and, therefore, do not provide a strict upper bound on the ridge yield. The present uncertainty on the background level also precludes a well-constrained measurement of width $\Delta \phi$, which has recently been proposed as a potential way to discriminate models for the origin of the ridge yield [32].

Based on these two independent estimates for the background level and shape, we conclude that a significant near-side ridge yield is present for $6<p_{t}^{\text {trig }}<10 \mathrm{GeV} / c$, indicating that the ridge is indeed correlated with jet production in central $\mathrm{Au}+\mathrm{Au}$ collisions.

\section{CHARACTERIZATION OF THE RIDGE, $p_{t}^{\text {assoc }}$ DEPENDENCE}

Figure 6 shows the $p_{t}$ spectrum of associated particles in the ridge, $Y_{\text {ridge }}$ (left) and the jet-like peak, $Y_{J}^{\Delta \eta}$ (right) [see Eqs. (9) and (8), respectively]. We only consider $p_{t}^{\text {trig }}>$ $4 \mathrm{GeV} / c$, where the jet-like peak as defined here is symmetric in $\Delta \eta$ and $\Delta \phi$ and the peak widths are similar to the $d+\mathrm{Au}$ reference measurements. Table III characterizes the spectra through their inverse slope parameter $T$ from the fit of an exponential function, $\frac{d N}{d p_{t}} \propto p_{t} e^{-p_{t} / T}$.

The jet-like spectrum is significantly harder than the inclusive spectrum and similar to the $d+\mathrm{Au}$ reference measurement, whereas the ridge spectrum is softer, though still harder than the inclusive spectrum. For $4<p_{t}^{\text {trig }}<$ $6 \mathrm{GeV} / c$, the normalized jet-like yield per trigger is similar in central $\mathrm{Au}+\mathrm{Au}$ and $d+\mathrm{Au}$ collisions, whereas at $6<$ $p_{t}^{\text {trig }}<10 \mathrm{GeV} / c$ the yield is slightly enhanced in $\mathrm{Au}+\mathrm{Au}$ collisions.

\section{DISCUSSION AND SUMMARY}

The similarity of peak shape and $p_{t}$ distribution of the jet-like yield in central $\mathrm{Au}+\mathrm{Au}$ and $d+\mathrm{Au}$ collisions, in contrast to the softer $p_{t}^{\text {assoc }}$ distribution and the approximately

TABLE III. Slope parameter $T$ from an exponential fit (see Fig. 6) to the $p_{t}^{\text {assoc }}$ spectrum in different $p_{t}^{\text {trig }}$ bins for ridge-like $\left(T_{\text {ridge }}\right)$ and jet-like $\left(T_{\text {jet }}\right)$ near-side correlations and $T_{\text {jet }}^{d \mathrm{Au}}$ for the $d+\mathrm{Au}$ reference measurement (statistical error only). The slope of the inclusive spectrum is $T=355 \pm 6 \mathrm{MeV} / c$.

\begin{tabular}{lccc}
\hline \hline$p_{t}^{\text {trig }}(\mathrm{GeV} / c)$ & $T_{\text {ridge }}(\mathrm{MeV} / c)$ & $T_{\text {jet }}(\mathrm{MeV} / c)$ & $T_{\text {jet }}^{d \text { Au }}(\mathrm{MeV} / c)$ \\
\hline $4-6$ & $416 \pm 22$ & $598 \pm 21$ & $647 \pm 24$ \\
$6-10$ & $514 \pm 148$ & $702 \pm 47$ & $723 \pm 86$ \\
\hline \hline
\end{tabular}



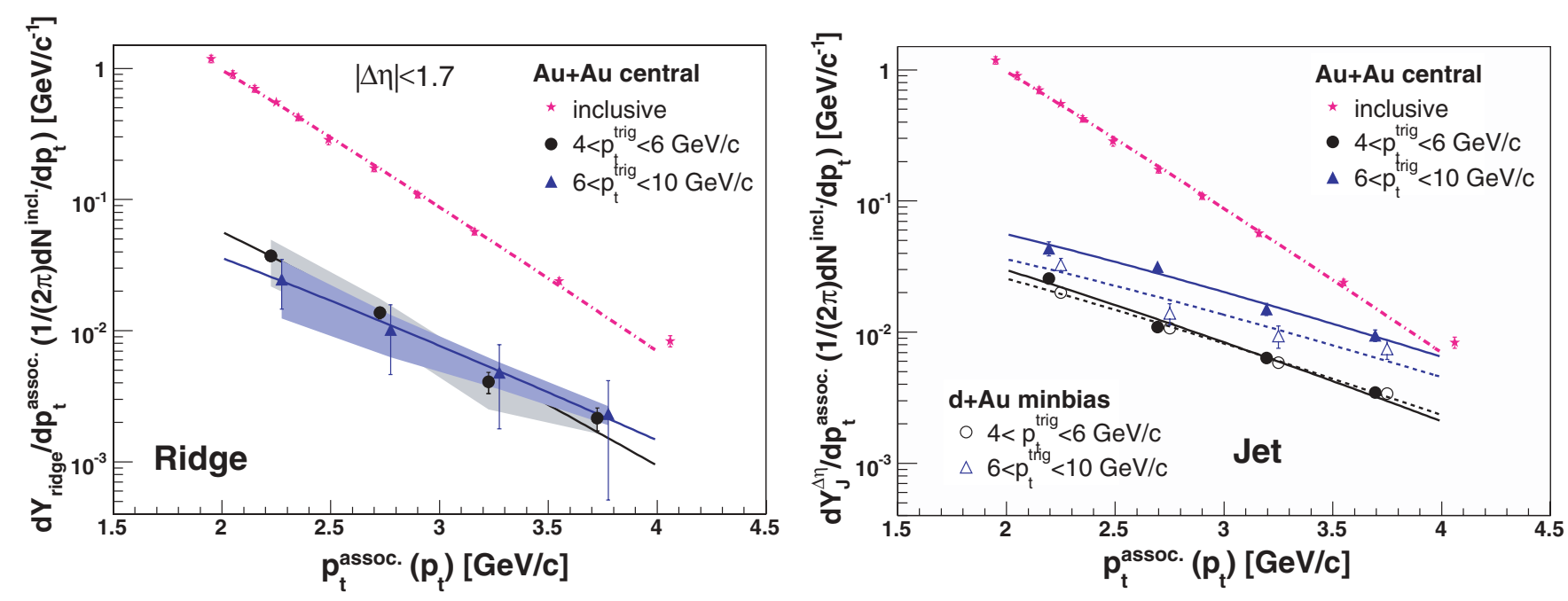

FIG. 6. (Color online) Differential $p_{t}$ spectrum for associated particles in central Au $+\mathrm{Au}$ collisions, with $4<p_{t}^{\text {trig }}<6$ and $6<p_{t}^{\text {trig }}<$ $10 \mathrm{GeV} / c$. The dash-dotted line is the inclusive hadron spectrum from central Au $+\mathrm{Au}$ collisions [1]. Left panel: ridge spectrum; shaded bands show systematic uncertainty. Right panel: jet-like spectrum, also compared to $d+$ Au reference measurements. The lines in both panels show exponential fits to the data (see Table III). Data are offset horizontally for clarity.

$\Delta \eta$-independent shape of the ridge yield in central $\mathrm{Au}+\mathrm{Au}$ collisions, supports the picture that the near-side correlation at high $p_{t}^{\text {trig }}$ in central $\mathrm{Au}+\mathrm{Au}$ collisions consists of two distinct components: a vacuum jet fragmentation contribution, similar to that seen in $p+p$ and $d+$ Au reference measurements, and the ridge contribution, with properties similar to bulk particle production.

Currently available models of ridge formation [18-26] provide only qualitative guidance about the underlying physics of the ridge, not quantitative predictions at sufficient precision to exclude a given picture based on the present measurements. All current models generate a softer spectrum for the ridge yield than for the jet-like associated yield and describe qualitatively the results in Fig. 6. The models involving turbulent color fields $[19,20]$ predict a broadening of the jetlike peak in $\Delta \eta$, which is not observed in these measurements at high $p_{t}$. The observed longitudinal extent of the ridge $(|\Delta \eta|>1.5)$ indicates qualitatively that the ridge is formed early in the evolution of the fireball (for example, as color flux tubes from a CGC initial state $[25,26]$ ) and disfavors the gluon radiation [18] and the turbulent color field mechanisms $[19,20]$, which invoke final-state partonic energy loss and (subsequent) coupling of the radiated gluons to the bulk matter or color fields. The momentum kick model [21] and the trigger bias model $[23,24]$ may accommodate the width of the ridge, though with assumptions about the momentum distribution and density of the thermal background and the radial flow boost of the underlying $p+p$ event that are not yet constrained by available data on inclusive yields and spectra, in addition to other correlation measurements. Another model attributes the ridge structure to heating of the medium and hadronization by quark recombination from QCD matter and seems to reproduce preliminary versions of the measurement [22] but does not treat longitudinal dynamics explicitly. We anticipate that the measurements of the correlation shapes and yields in this article should lead to a reassessment of the various models and a more quantitative confrontation of the models with the measurements.

In summary, the analysis of di-hadron $\Delta \eta \times \Delta \phi$ correlations in central $\mathrm{Au}+\mathrm{Au}$ collisions reveals a more complex structure of the near-side correlation than that expected from $p+p$ and $d+$ Au reference measurements, namely the observation of additional correlated yield at large $\Delta \eta$ (the ridge). New detailed measurements of the shape and the $p_{t}^{\text {trig }}$ and $p_{t}^{\text {assoc }}$ dependence of the ridge- and jet-like contributions support the picture that the near-side two-particle correlation consists of two distinct components: a $\Delta \eta$-independent ridge contribution with properties similar to inclusive particle production and a jet contribution similar to that seen in $p+p$ and $d+\mathrm{Au}$ reference measurements. Various mechanisms have already been proposed for the formation of the ridge in heavy-ion collisions. The measurements presented here are expected to rule out or constrain some of the proposed models.

\section{ACKNOWLEDGMENTS}

We thank the RHIC Operations Group and RCF at BNL, and the NERSC Center at Lawrence Berkeley National Laboratory and the resources provided by the Open Science Grid consortium for their support. This work was supported in part by the Offices of NP and HEP within the US Department of Energy Office of Science, the US National Science Foundation, the Sloan Foundation, the DFG cluster of excellence "Origin and Structure of the Universe"; CNRS/IN2P3, RA, RPL, and EMN of France; STFC and EPSRC of the United Kingdom; FAPESP of Brazil; the Russian Ministry of Science and Technology; the NNSFC, CAS, MoST, and MoE of China; IRP and GA of the Czech Republic; FOM of the Netherlands; DAE, DST, and CSIR of the Government of India; the Polish State Committee for Scientific Research; and the Korea Sci. \& Eng. Foundation. 
[1] J. Adams et al. (STAR Collaboration), Phys. Rev. Lett. 91, 172302 (2003).

[2] S. S. Adler et al. (PHENIX Collaboration), Phys. Rev. Lett. 91, 072301 (2003).

[3] C. Adler et al. (STAR Collaboration), Phys. Rev. Lett. 90, 082302 (2003).

[4] J. Adams et al. (STAR Collaboration), Phys. Rev. Lett. 95, 152301 (2005).

[5] J. Adams et al. (STAR Collaboration), Phys. Rev. Lett. 97, 162301 (2006).

[6] J. Adams et al. (STAR Collaboration), Nucl. Phys. A757, 102 (2005).

[7] K. Adcox et al. (PHENIX Collaboration), Nucl. Phys. A757, 184 (2005).

[8] I. Arsene et al. (BRAHMS Collaboration), Nucl. Phys. A757, 1 (2005).

[9] B. B. Back et al. (PHOBOS Collaboration), Nucl. Phys. A757, 28 (2005).

[10] J. Adams et al. (STAR Collaboration), Phys. Rev. C 73, 064907 (2006).

[11] J. Adams et al. (STAR Collaboration), Phys. Rev. C 75, 034901 (2007).

[12] J. Adams et al. (STAR Collaboration), Phys. Rev. C (to be published), nucl-ex/0601042.

[13] B. I. Abelev et al. (STAR Collaboration), Phys. Rev. Lett. 97, 152301 (2006).

[14] R. J. Fries, B. Muller, C. Nonaka, and S. A. Bass, Phys. Rev. C 68, 044902 (2003).

[15] R. C. Hwa and C. B. Yang, Phys. Rev. C 67, 034902 (2003).
[16] R. C. Hwa and C. B. Yang, Phys. Rev. C 70, 054902 (2004).

[17] B. Alver et al. (PHOBOS Collaboration), Phys. Rev. Lett. (to be published), arXiv:0903.2811 [nucl-ex].

[18] N. Armesto, C. A. Salgado, and U. A. Wiedemann, Phys. Rev. Lett. 93, 242301 (2004).

[19] P. Romatschke, Phys. Rev. C 75, 014901 (2007).

[20] A. Majumder, B. Muller, and S. A. Bass, Phys. Rev. Lett. 99, 042301 (2007).

[21] C. Y. Wong, Phys. Rev. C 76, 054908 (2007).

[22] C. B. Chiu and R. C. Hwa, Phys. Rev. C 72, 034903 (2005).

[23] S. A. Voloshin, Phys. Lett. B632, 490 (2006).

[24] E. V. Shuryak, Phys. Rev. C 76, 047901 (2007).

[25] A. Dumitru, F. Gelis, L. McLerran, and R. Venugopalan, Nucl. Phys. A810, 91 (2008).

[26] S. Gavin, L. McLerran, and G. Moschelli, Phys. Rev. C 79, 051902(R) (2009).

[27] K. H. Ackermann et al. (STAR Collaboration), Nucl. Instrum. Methods A 499, 624 (2003).

[28] J. Adams et al. (STAR Collaboration), Phys. Rev. Lett. 91, 072304 (2003).

[29] N. N. Ajitanand, J. M. Alexander, P. Chung, W. G. Holzmann, M. Issah, R. A. Lacey, A. Shevel, A. Taranenko, and P. Danielewicz, Phys. Rev. C 72, 011902(R) (2005).

[30] S. S. Adler et al. (PHENIX Collaboration), Phys. Rev. Lett. 97, 052301 (2006).

[31] J. Adams et al. (STAR Collaboration), Phys. Rev. C 72, 014904 (2005)

[32] J. L. Nagle, Nucl. Phys. A830, 147c (2009). 